\title{
Quality of life assessment in patients with HNF1A-MODY and GCK- MODY
}

\author{
Magdalena Szopa $\mathbb{1}^{1,2} \cdot$ Bartlomiej Matejko ${ }^{1,2} \cdot$ Damian Ucieklak ${ }^{1,2} \cdot$ Agata Uchman $^{1} \cdot$ Jerzy Hohendorff ${ }^{1,2}$. \\ Sandra Mrozińska ${ }^{1,2} \cdot$ Wojciech Głodzik ${ }^{3}$ - Barbara Zapała ${ }^{4}$. Teresa Płatek ${ }^{4}$ Iwona Solecka ${ }^{2}$ Cyrus M Sani ${ }^{1}$. \\ Maciej T. Małecki ${ }^{1,2}$
}

Received: 31 August 2018 / Accepted: 1 November 2018 / Published online: 12 November 2018

(c) Springer Science+Business Media, LLC, part of Springer Nature 2018

\begin{abstract}
Aim The impact of maturity onset diabetes of the young (MODY) on quality of life (QoL) has never been examined. We assessed disease impact on QoL among patients with HNF1A-MODY and GCK mutation carrier status.

Methods The study included 80 patients with HNF1A-MODY and 89 GCK gene mutation carriers. We also examined 128 type 1 diabetes (T1DM) patients for comparison. Diabetes-specific QoL was assessed using the Audit of Diabetes Dependent Quality of Life questionnaire.

Results HNF1A-MODY and GCK-MODY groups had similar mean age (41.7 vs. 38.0 years, respectively) and BMI (24.1 vs. $24.3 \mathrm{~kg} / \mathrm{m}^{2}$ ), whereas T1DM patients were on average younger (34.2 years) with similar BMI $\left(25.0 \mathrm{~kg} / \mathrm{m}^{2}\right)$. Less than a third of GCK mutation carriers were on pharmacotherapy $(n=20,31 \%)$, while the majority of $H N F 1 A$ mutation carriers used oral drugs or insulin $(n=66,82.5 \%)$. While current QoL was similar across the three groups $(p=0.66)$, two other major indices - the impact of diabetes on QoL and the average weighted impact (AWI)—differed among them $(p<0.001$ for both comparisons). The impact of diabetes on patient QoL and AWI observed in both MODY groups was smaller than in T1DM. Etiological diagnosis of diabetes and a diagnosis of retinopathy were the only independent factors influencing the impact of diabetes on QoL and AWI in regression analysis. In HNF1A-MODY, all three major indices of QoL were more heavily influenced for patients on insulin in comparison to other treatment sub-groups.

Conclusion MODY has a smaller negative impact on QoL compared to T1DM. Mode of treatment further stratifies QoL decline for HNF1A-MODY subjects.
\end{abstract}

Keywords Diabetes $\cdot$ MODY $\cdot$ Quality of life

\section{Introduction}

Quality of life (QoL) is a well-established concept; however, it does not possess a commonly accepted definition. According to the World Health Organization (WHO), QoL

Maciej T. Małecki

malecki_malecki@yahoo.com

maciej.malecki@uj.edu.pl

1 Department of Metabolic Diseases, Jagiellonian University Medical College, Krakow, Poland

2 University Hospital, Krakow, Poland

3 Sanatio Medical Center, Krakow, Poland

4 Department of Clinical Biochemistry, Jagiellonian University Medical College, Krakow, Poland consists of an individual's perceptions of one's life in the context of value systems, culture, concerns and individual ambitions [1]. QoL is also affected by factors such as psychological well-being, physical health and fitness, independence, relationships with other people, and the environment in which the person lives [2]. In the case of patients with diabetes, specific QoL indices are used to create a comprehensive, precise, and personal assessment of the disease along with its role in the individual's life [3].

A diagnosis of either major form of this disease-type 1 diabetes (T1DM) and type 2 diabetes (T2DM) - is associated with deterioration in QoL [3-6]. However, no QoL data are available for patients with monogenic diabetes which has an estimated prevalence of $2-5 \%$ of all diabetic patients [7]. MODY is a heterogeneous group of singlegene diabetes. A single mutation in either the $G C K$ or HNF1A gene accounts for up to $80 \%$ of all MODY cases, 
and presents as a diverse clinical picture. Individuals with GCK-MODY are born with a mildly elevated glucose level that neither requires pharmacotherapy nor progresses substantially later in life [8]. In contrast, HNF1A-MODY patients develop diabetes in the 2 nd and 3rd decades of life, and usually need pharmacological treatment to both control hyperglycemia and subsequently prevent chronic diabetic complications [9]. Sulfonylurea (SU) tablets are particularly effective in controlling glucose level in HNF1A-MODY; however, insulin therapy may be necessary later in life [9, 10]. Although MODY is generally a less severe form of the disease as compared to T1DM, its diagnosis is also likely to impact QoL for affected individuals. This can be further influenced by the fact that many MODY patients receive unnecessary or inadequate treatment, sometimes in spite of molecular diagnosis [10].

In this study, we aimed to assess a health-related QoL estimate in patients with a genetic diagnosis of HNF1AMODY and $G C K$ mutation carrier status. We subsequently sought to compare this estimate with that of T1DM patients by using the Audit of Diabetes Dependent Quality of Life (ADDQoL) questionnaire.

\section{Patients and methods}

We attempted to contact all adult patients who had been registered in our MODY patient database over approximately the last 15 years [11]. We initially considered 130 diabetic patients with HNF1A-MODY and 128 subjects with GCK-MODY mutations. Eventually, 80 HNF1AMODY and 89 GCK-MODY patients (45 diabetic, 44 pre-diabetic) completed the questionnaire. The rest of the potential participants from the database either could not be reached-using the contact information they had provided us - or, rarely, refused to participate in the study. The basic characteristics, such as age and gender, of MODY individuals who did not enter the study were not different from the actual study participants as discussed below. We also included 128 consecutive T1DM patients from two diabetes outpatient clinics for the comparison.

The patients were considered eligible for this study if they were previously diagnosed with diabetes, were on hypoglycemic therapy, or met the WHO criteria based on fasting glucose measurements at the initial examination. For GCK-MODY, we also included mutation carriers with formal fasting pre-diabetic status as they were generally informed by their physicians to be affected by a genetically inherited form of diabetes. All study individuals were white Caucasian residents of southeastern Poland. The MODY participants previously had a genetic diagnosis of MODYrelated mutation established during research activities performed at the Department of Metabolic Diseases,
Jagiellonian University Medical College in Krakow, Poland. The pathogenicity of mutations was determined based on earlier published reports, DNA sequencedifference biological character, and co-segregation with diabetes within the families. The ascertainment criteria and genetic testing procedures for MODY were as previously described [11]. Subjects were defined as having T1DM if they had typical clinical symptoms at diagnosis, an insulin therapy requirement from the beginning of the disease, and were diagnosed prior to the age of 30 with diabetes. They were recruited from two out-patient clinics in Krakow.

Subjects received a standard questionnaire and subsequently underwent physical examination. Diagnosis of diabetes complications was based on questionnaires. We excluded all individuals with severe concomitant chronic diseases of the respiratory tract, liver, or kidney. Additionally, pregnant women, as well as patients with neoplasms or active infections were also screened out of the study. Laboratory and genetic analyses were performed as reported in our previous papers [12]. This study was performed in compliance with the Helsinki Declaration, and its design was reviewed and subsequently approved by the university Bioethical Committee.

Diabetes specific QoL was assessed using the latest, 19th Polish edition of the ADDQoL [13], which determines an individual's perception and significance of the impact of diabetes on various aspects of their QoL [14]. This Polish version of the ADDQoL was professionally validated. Participants evaluated the impact of the disease on different domains of their life using a scale from -3 (maximum negative impact) to +1 (maximum positive impact). Further, they ranked the weight of the domain for their QoL on a scale from 3 (very important) to 0 (not at all important) [15]. Ranging from -9 (maximum negative impact) to +3 (maximum positive impact), the weighted impact score for every domain was calculated by multiplying the impact rating by the importance rating [14]. To determine an overall continuous Average Weighted Impact (AWI) score, we summarized the weighted ratings of relevant domains, and then divided the result by the number of domains http://www.r-project.org. A low continuous ADDQoL score indicates a negative impact of the disease on QoL. We performed statistical analyses to determine the difference between the two (student $t$-test) and three (ANOVA) study groups; non-parametric tests were applied as equivalents (Mann-Whitney $U$-test; Kruskal-Wallis test) where necessary. For post hoc analysis, pairwise Wilcox test with Bonferroni correction was applied. We used chi-square or Fisher's exact tests where appropriate for categorical variables. Additionally we fit the multivariate regression models to identify significant predictors of QoL indicators (present QoL, impact of diabetes, AWI) from among 6 independent possible cofounders: time from diabetes diagnosis, age of 
Table 1 Clinical characteristics of patients with HNF1AMODY, GCK-MODY and T1DM

\begin{tabular}{|c|c|c|c|c|c|}
\hline & $\begin{array}{l}\text { HNF1A- } \\
\text { MODY }\end{array}$ & $\begin{array}{l}\text { GCK- } \\
\text { MODY }\end{array}$ & T1DM & $p$ & Post hoc \\
\hline$N$ & 80 & 89 & 128 & & - \\
\hline Gender $[\mathrm{F} / \mathrm{M}]$ & $56 / 24$ & $52 / 37$ & $61 / 67$ & 0.006 & - \\
\hline Age at the examination [years] & $41.7 \pm 14.8$ & $38.0 \pm 14.2$ & $\begin{array}{l}34.2 \pm \\
12.1\end{array}$ & 0.001 & $\sim$ \\
\hline Diabetes duration [years] & $18.8 \pm 11.9$ & $10.0 \pm 7.9$ & $15.4 \pm 9.8$ & $<0.001$ & $* \wedge$ \\
\hline Age at which diabetes was diagnosed [years] & $24.1 \pm 10.1$ & $27.9 \pm 14.1$ & $\begin{array}{l}19.2 \pm \\
10.4\end{array}$ & $<0.001$ & $\wedge_{\sim}$ \\
\hline Years since MODY genetic diagnosis [years] & $4.7 \pm 3.3$ & $3.1 \pm 2.1$ & - & 0.002 & - \\
\hline $\begin{array}{l}\text { Current treatment : no pharmacotherapy/ oral } \\
\text { drugs/insulin with insulin }[N / N / N]\end{array}$ & $14 / 38 / 28$ & $69 / 15 / 5$ & $0 / 0 / 128$ & $<0.001$ & - \\
\hline Body Mass Index $\left[\mathrm{kg} / \mathrm{m}^{2}\right]$ & $24.1 \pm 3.8$ & $24.3 \pm 3.7$ & $25.0 \pm 3.7$ & 0.168 & - \\
\hline $\mathrm{HbA1c}[\%]$ & $6.8 \pm 1.5$ & $6.3 \pm 0.6$ & $7.3 \pm 1.2$ & $<0.001$ & $\wedge \sim$ \\
\hline Retinopathy [Yes/No] & $20 / 60$ & $1 / 87$ & $26 / 101$ & $<0.001$ & - \\
\hline Nephropathy [Yes/No] & $5 / 75$ & $0 / 89$ & $9 / 118$ & 0.041 & - \\
\hline Neuropathy [Yes/No] & $17 / 63$ & $3 / 86$ & $17 / 110$ & 0.002 & - \\
\hline Cardiovascular Disease [Yes/No] & $16 / 64$ & $6 / 83$ & $7 / 119$ & 0.001 & - \\
\hline $\begin{array}{l}\text { Hypertension }[\mathrm{No} / \text { Yes, without treatment/ } \\
\text { Yes, on hypertensive treatment] }\end{array}$ & $50 / 6 / 24$ & $66 / 4 / 19$ & $106 / 0 / 20$ & 0.003 & - \\
\hline Smoking status [Never/in the past/ currently] & $49 / 18 / 13$ & $55 / 20 / 13$ & $110 / 8 / 8$ & $<0.001$ & - \\
\hline
\end{tabular}

MDI multiple daily injection, CSII continuous subcutaneous insulin infusion $p<0.05$ : HNF1A vs. GCK* ; GCK vs. T1DM^ ; T1DM vs. HNF1A examination, BMI, gender, presence of retinopathy, and type of diabetes diagnosis (with T1DM as reference). $P$ values less than 0.05 were considered significant. Statistical analysis was carried out using $\mathrm{R}$ software version 3.4 .2 [16].

\section{Results}

The clinical characteristics of the study groups are presented in Table 1. More women than men responded in both MODY cohorts, whereas in T1DM slightly more men were included. HNF1A-MODY patients and GCK-MODY mutation carriers had similar age at examination (41.7 \pm 14.8 years vs. $38.0 \pm 14.2$ years, respectively) and BMI $\left(24.1 \pm-15.4 \mathrm{~kg} / \mathrm{m}^{2}\right.$ vs. $\left.24.3 \pm 3.7 \mathrm{~kg} / \mathrm{m}^{2}\right)$. T1DM patients were similar in age to GCK mutation carriers but significantly younger ( $34.2 \pm 12.1$ years) than HNF1A-MODY patients, and had BMI similar $\left(25.0 \mathrm{~kg} / \mathrm{m}^{2}\right)$ to both MODY groups. Less than a third of $G C K$ mutation carriers were on pharmacotherapy $(n=20,31 \%)$, while the majority of HNF1A mutation carriers used oral drugs or insulin $(n=66$, 82.5\%). Among those patients with HNF1A-MODY who were on pharmacotherapy, more than 50\% used SU $(n=$ $44)$, around $15 \%$ used metformin $(n=13)$, and almost $40 \%$ were on insulin therapy $(n=20)$. All T1DM patients were on intensive insulin therapy: exactly half of them used insulin pumps, while the others were on multiple daily injections. There was a higher proportion of diabetic complications in both the HNF1A-MODY and the T1DM groups than in the GCK mutation carriers (Table 1).

There was no difference in current QoL between the three groups: $1.15 \pm 0.70$ vs. $1.23 \pm 0.76$ vs. $1.14 \pm 0.74$, respectively, in HNF1A-MODY and GCK-MODY and T1DM $(p=0.66)$ (Fig. 1). However, both AWI and the impact of diabetes on the patient's QoL differed between the groups $(p<0.001 ; p<0.001)$. The impact of diabetes on the patient's QoL was the largest in T1DM patients $(-1.6 \pm$ 1.05). Less profound impacts were identified in HNF1AMODY $(-0.96 \pm 1.05 ; p<0.05)$, as well as in GCKMODY $(-0.63 \pm 0.88 ; p<0.001)$. Similarly, group AWI decreased most in T1DM $(-1.99 \pm 1.39)$ followed by HNF1A-MODY $(-1.52 \pm 1.44 ; p<0.05)$ and then GCKMODY $(-0.95 \pm 1.06 ; p<0.001)$. Both the impact of diabetes and AWI indices were worse in the HNF1A group as compared to the GCK-MODY cohort $(p<0.05$ for both comparisons).

Additional analyses were performed according to the treatment method in HNF1A-MODY, for which different therapeutic approaches are used. Pharmacotherapy (insulin and/or OHA) had a significant impact on QoL. When comparing patients on insulin $(n=30)$ with the rest of MODY group $(n=50)$, all three major indices-'present QoL', 'impact of diabetes on QoL' and AWI-were worse in the insulin group ( $\mathrm{p}$ values of $0.02,0.000$, and 0.003 , respectively) (Table $2 \mathrm{~A})$. 
Fig. 1 Diabetes mean impact on QoL in patients with HNF1AMODY, GCK-MODY, and T1DM

Table $2 \mathrm{QoL}$ in relation to method of treatment

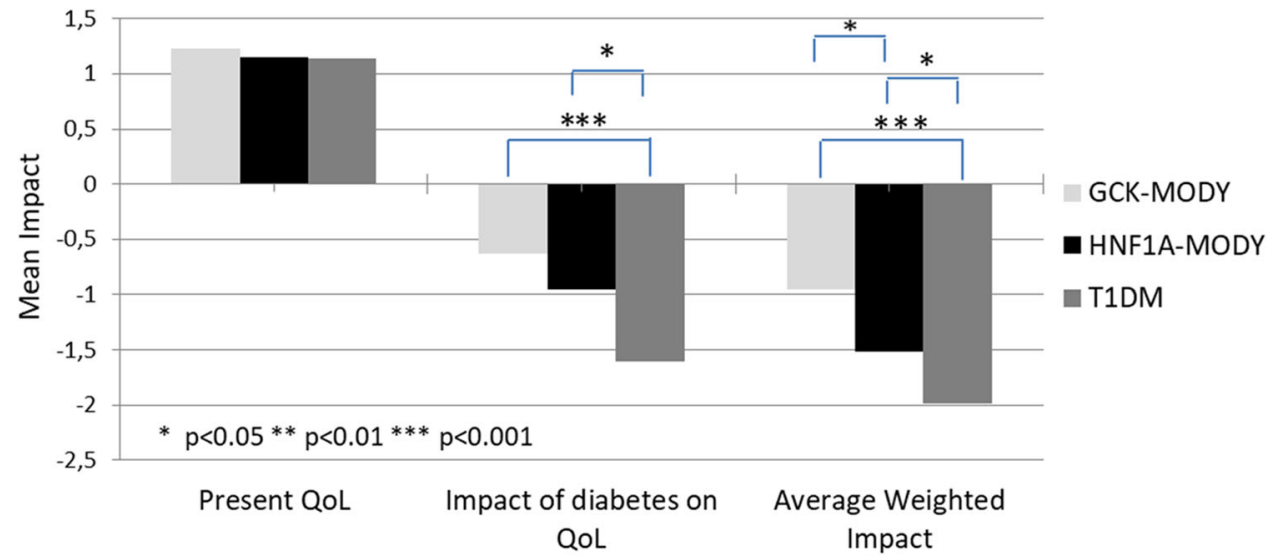

\begin{tabular}{|c|c|c|c|c|c|c|}
\hline \multicolumn{7}{|l|}{ A. HNF1A-MODY } \\
\hline & \multicolumn{2}{|c|}{ Present QOL } & \multicolumn{2}{|c|}{$\begin{array}{l}\text { Impact of diabetes on } \\
\text { QoL }\end{array}$} & \multicolumn{2}{|c|}{$\begin{array}{l}\text { Average weighted } \\
\text { impact }\end{array}$} \\
\hline \multicolumn{7}{|l|}{ HNF1A-MODY } \\
\hline On insulin $(n=30)$ & 0.9 & $P=0.02$ & -1.65 & $P<0.0001$ & -2.33 & $P<0.001$ \\
\hline Other $(n=50)$ & 1.3 & & -0.56 & & -1.03 & \\
\hline \multicolumn{7}{|l|}{ HNF1A-MODY } \\
\hline On diet only $(n=14)$ & 1.5 & $P=0.03$ & -0.36 & $P=0.01$ & -0.64 & $P<0.01$ \\
\hline On pharmacotherapy $(n=66)$ & 1.08 & & -1.09 & & -1.71 & \\
\hline \multicolumn{7}{|l|}{ B. GCK-MODY } \\
\hline & \multicolumn{2}{|c|}{ Present QOL } & \multicolumn{2}{|c|}{$\begin{array}{l}\text { Impact of diabetes on } \\
\text { QoL }\end{array}$} & \multicolumn{2}{|c|}{$\begin{array}{l}\text { Average weightened } \\
\text { impact }\end{array}$} \\
\hline \multicolumn{7}{|l|}{ GCK-MODY } \\
\hline Diabetic $(n=45)$ & 1.08 & $P=0.12$ & -0.54 & $P=0.447$ & -0.9 & $P=0.46$ \\
\hline Prediabetic $(n=44)$ & 1.36 & & -0.73 & & -1.0 & \\
\hline \multicolumn{7}{|l|}{ GCK-MODY } \\
\hline On diet only $(n=69)$ & 1.32 & $P=0.06$ & -0.63 & $P=0.65$ & -0.91 & $\mathrm{P}=0.33$ \\
\hline On pharmacotherapy $(n=20)$ & 0.95 & & -0.66 & & -1.0 & \\
\hline
\end{tabular}

No significant subgroup differences were found for diabetes status or method of treatment in the GCK-MODY group across all three major QoL indices (Table 2B). Additionally, no gender difference were found both within the status and treatment subgroups, as well as over the entire cohort across all three QoL indices.

Regression models were also built for the main QoL indices to search for significant independent factors. Age of examination was the only independent predictor of the current QoL $(p<0.001 ; b=-0.016)$. Regarding the impact of diabetes on QoL, a diagnosis of retinopathy $(p=0.028$; $b=-0.53)$ and the difference between GCK-MODY and T1DM and between HNF1A-MODY and T1DM $(p<$ $0.0001 ; b=0.849, p<0.001 ; b=0.571)$ were independent influencing factors. The multiple regression model with
AWI as the dependent variable again showed the above variables (the presence of retinopathy $-p=0.028, b=$ -0.53 ; the difference between GCK-MODY and T1DM $-p<0.0001 ; b=1.009$, and between HNF1A-MODY and T1DM $p=0.013 ; b=0.525)$ as predictors of average weighted impact. No other analyzed variables were found significant in the models.

When specific domains of QoL in three main groups were analyzed, we found differences in most -14 out of 19 - domains (Fig. 2). The only non-significant finding were in 'freedom to eat', 'freedom to drink', physical appearance', 'people's reaction', and 'living conditions' domains. In terms of absolute values, T1DM was characterized by the highest impact in 14 domains except 'freedom to eat', 'freedom to drink', 'motivation', 'physical appearance', 
'family life', 'people's reaction'. Conversely, GCK-MODY was characterized by the lowest impact on specific domains except 'freedom to eat' (slightly higher impact than in HNF1A-MODY).

We also compared patients on insulin from two different groups (HNF1A-MODY and T1DM) to search for differences in specific domains (Figs. 3 and 4). Surprisingly, absolute negative impact was higher in HNF1A-MODY than in T1DM for the majority of the domains (15 out of 19), with the differences being significant in three: 'freedom to eat', 'motivation', and 'family life' (Fig. 3). Diabetes impact on specific domains of QoL in patients with HNF1A-MODY according to treatment method, insulin vs. all the others therapies, is shown in Fig. 4.

\section{Discussion}

Our literature search indicates our work to be the first report of disease impact on QoL for patients with either of the two most common forms of monogenic diabetes: HNF1AMODY and GCK-MODY. We observed a substantial impact by diabetes on QoL in both single gene disease groups. We also compared data from both MODY subtypes with T1DM, and described differences between cohorts of patients related to both etiological diagnosis, as well as mode of treatment.

Being diagnosed with T1DM or T2DM diabetes has previously been reported to have an important impact on QoL: the disease usually changes the person's psychological comfort, lifestyle, and general well-being [3-6]. These

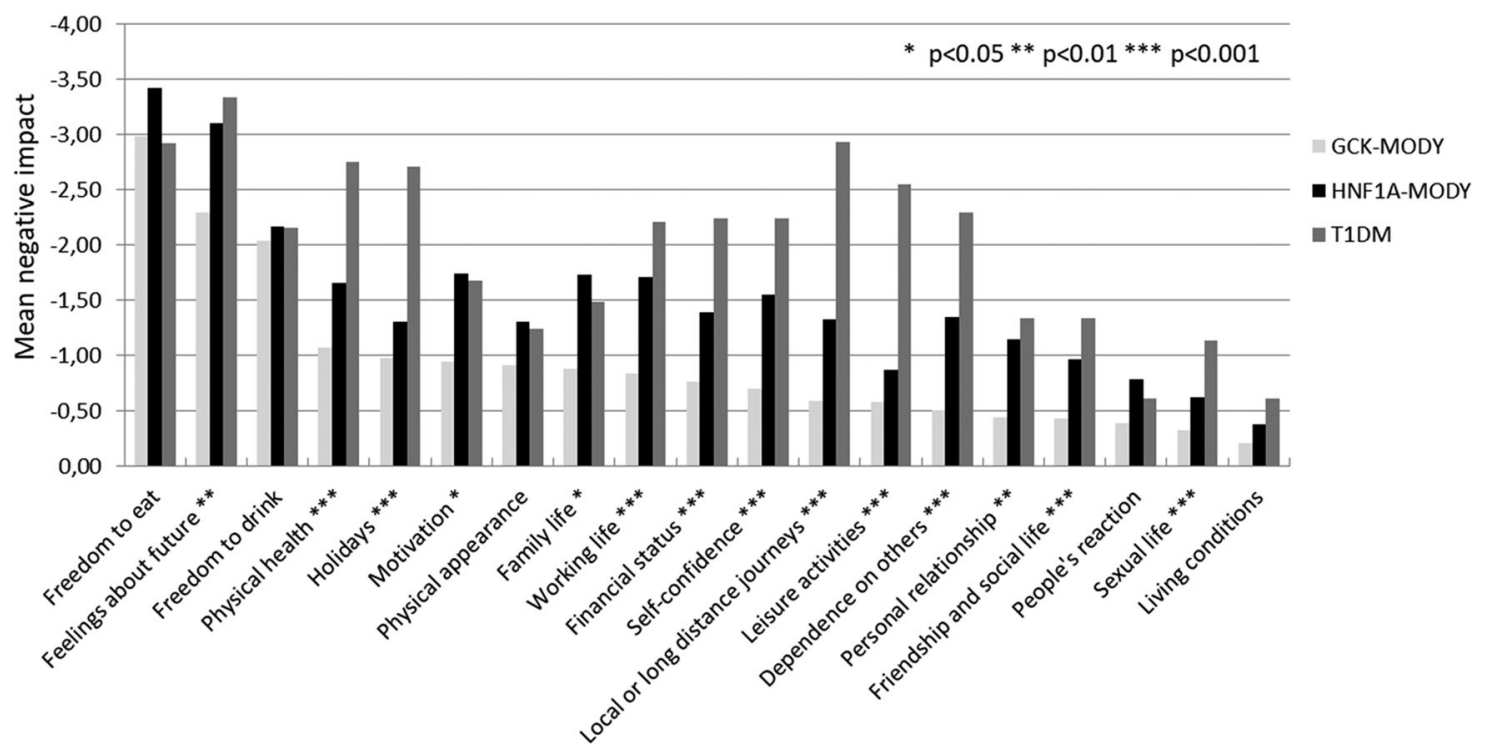

Fig. 2 Diabetes mean impact on specific domains of QoL in patients with HNF1A-MODY GCK-MODY and T1DM

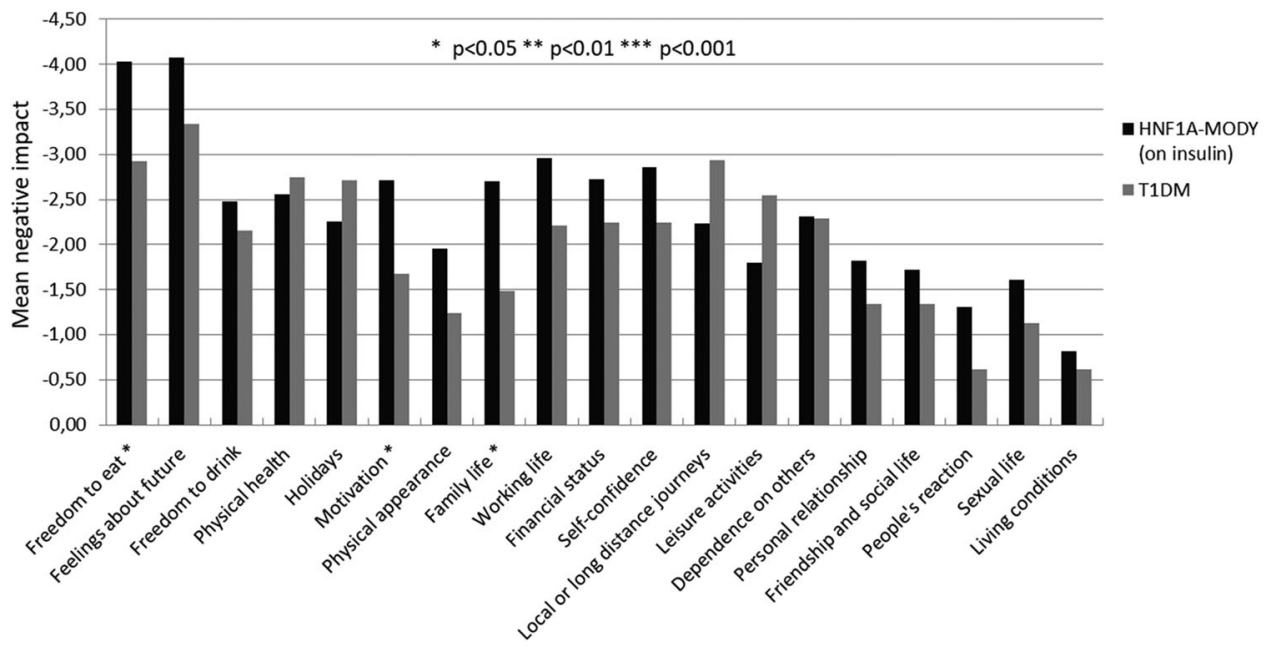

Fig. 3 Diabetes mean impact on specific domains of QoL in patients with HNF1A-MODY compared to T1DM 
Fig. 4 Diabetes mean impact on specific domains of QoL in patients with HNF1A-MODY according to treatment method

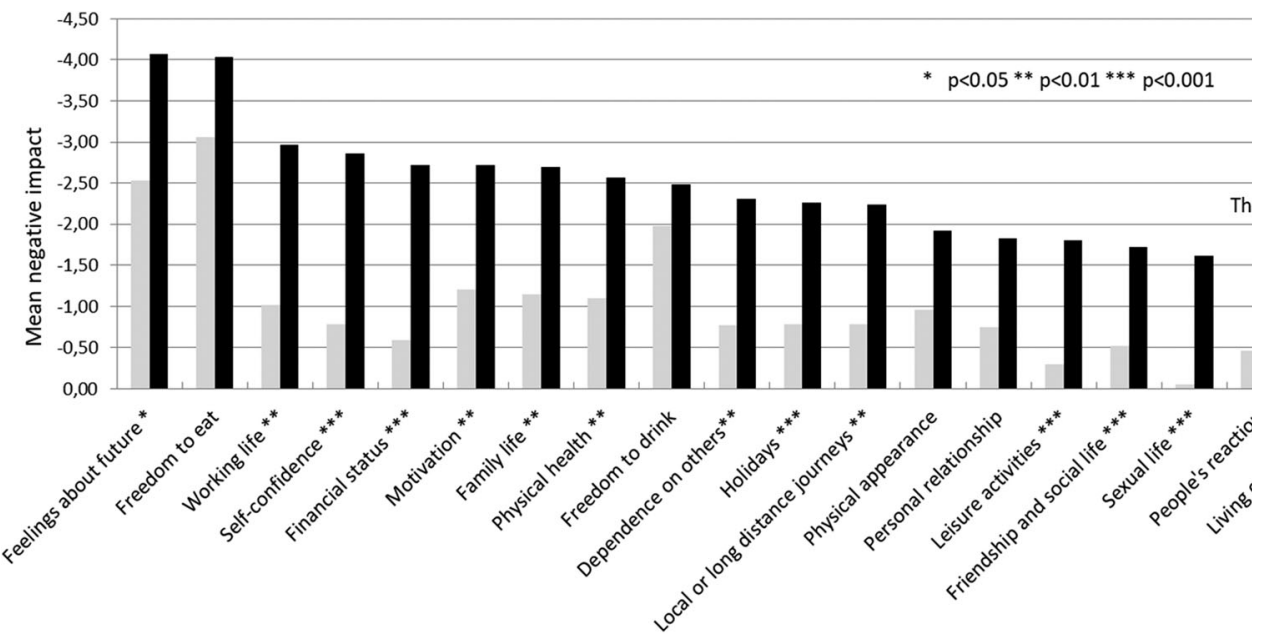

changes frequently lead to feelings of losing control accompanied by an increase in both stress and emotional sensitivity. The results of the Diabetes Attitudes, Wishes, and Needs (DAWN) study showed that more than $40 \%$ of patients with T1DM and T2DM had impaired QoL and diabetes contributed to many psychosocial problems [16].

In the current study, the impact of diabetes on QoL and AWI was most significant among T1DM patients, followed by those in the HNF1A-MODY and then GCK-MODY group. These unsurprising differences are in line with wellknown disparities in the clinical picture among GCKMODY, HNF1A-MODY, and T1DM. Notably, etiological diagnosis together with a diagnosis of retinopathy wasthe only independent factor in our regression analysis influencing the impact of diabetes on QoL and AWI. We did not include a comparison group with T2DM from the Polish population, so a direct comparison was not possible. However, in a recently published study involving Spanish T2DM patients, the impact of diabetes on QoL seemed to be larger than in all our study groups [17]. The list of possible factors contributing to this fact includes age, frequent diabetes complications, and comorbidities.

Additionally, QoL in HNF1A-MODY - the more severe form of the two examined single gene disease-was better for patients being treated solely with diet-modification than in those on pharmacotherapy. Interestingly, insulin use in HNF1A-MODY was associated with a particularly large negative impact, showing a clear burden on QoL—probably even higher than in T1DM. Our findings concur with other research works where insulin use was associated with decreased QoL [18-21]. The reason for the significant impact of insulin use on QoL in HNF1A-MODY is not clear. One possible explanation is that T1DM patients are using insulin from the very beginning of the disease, while MODY individuals usually have to switch from oral hypoglycemic agents-which are generally more convenient to use.
In the current study, the largest impact of diabetes in all groups was observed on the 'freedom to eat', 'feelings about future', and 'freedom to drink' dimensions. Similar results were reported in earlier studies involving patients with both types of diabetes [13, 22-24]. The negative impact from the loss of dietary flexibility on the patients' diabetes-specific QoL refers not only to dietary restrictions around healthy eating and weight loss, but also the need to regularly monitor the relationship between food intake, energy expenditure, and blood glucose level for the majority of patients with diabetes.

A positive family history of diabetes is a typical feature of MODY. Interestingly, it was earlier reported that among patients with T2DM younger than 60 years of age, a positive history of diabetes was a protective factor for the quality of age [21]. This may be related to pre-existing information about diabetes and knowledge-sharing by family members affected by diabetes with those not yet affected. On the other hand, the AWI of diabetes on QoL was found in the same study to be more significantly negative in adults with early T2DM compared with those diagnosed later [25]. Early age of diagnosis is a typical feature of patients with MODY. Therefore, the balancing effect between younger age of diagnosis and positive family history of diabetes seems to be likely in MODY. The information provided by medical personnel to patients on their specific type of MODY diagnosis might have also been a factor influencing our results. Those with $G C K$ mutation were informed about both the mild nature of the defect and the unlikelihood of advanced diabetic complication risks among family members. This contrasts with the situation of patients diagnosed with HNF1A-MODY, who were warned about disease progression and the likely need for pharmacological treatment. Such counseling is likely to influence the QoL of the patient. It is interesting that in spite of the very moderate nature of the glycemic defect, rare pharmacological treatment requirement, and excellent 
prognosis, a substantial negative impact of diabetes on $\mathrm{QoL}$ was observed also in GCK-MODY. This seems to underline the importance of properly conveying information to patients by their consulting geneticists and diabetes specialists. Of note, some patients in the GCK-MODY group continued with pharmacological treatment after both the patient and their physician were informed about the nature of their genetic diagnosis. The disregarding of monogenic diabetes treatment guidelines for genetically tested GCKMODY patients by some physicians has been reported also in other countries-such as the UK, where $22 \%$ of GCKMODY patients were on pharmacological treatment after molecular diagnosis had been made [26].

The limitations of our study include the lack of pregenetic and post-genetic-diagnosis comparison-due to the post molecular-testing collection of the QoL results. Additionally, individuals with pre-diabetes were also included in the GCK-MODY group. Also, diagnosis of diabetic complications was based on questionnaires and not on medical examinations constituting a part of the study protocol. Moreover, variations in gender distribution were present across the study groups - the majority of patients were women in both MODY groups but not in T1DM. This variation may be related to the greater propensity of mothers to participate in the study due to intristic personal motivations regarding the hereditary, multigenerational nature of the disease. Instead, participation in the T1DM cohort was consecutive resulting in a balanced participation of both genders. It should be noted, however, we found no gender effect across and within our study groups. Furthermore, we did not systematically collect data on education and occupation of our study patients; thus, any sub-analysis based on these data was not possible. The study results could have been influenced by various potentially confounding factors that were not included in our regression analysis. Finally, we did not include patients with T2DM from our population for a direct comparison.

In summary, a diagnosis of HNF1A-MODY or GCKMODY, the most frequent subtypes of monogenic diabetes, has a negative impact on QoL for affected individuals. Mode of treatment seems to additionally influence QoL for HNF1A-MODY subjects. Educational programs with the objective of minimizing the negative impact of MODY on patient QoL are highly recommended in view of these findings.

Acknowledgements The authors would like to thank the patients who participated in the study. Additionally, the authors would like to thank also Ms. Agata Porębska for her technical assistance.

Funding The study was funded by grants from the Diabetes Poland Grant 2016, and the Jagiellonian University Medical College Grant (K/ ZDS/006219) awarded to Dr. Magdalena Szopa.
Author contributions M.S., D.U., A.U., J.H., S.M., W.G., B.Z., T.P., I.S., C.S. performed the research. M.S., B.M. analyzed data. M.S., M. T.M. designed the research study and interpreted the data. M.S., M.T. M. wrote the paper. M.T.M. is the guarantor of this work. All authors contributed to critical revision of the manuscript and approved its publication.

\section{Compliance with ethical standards}

Conflict of interest The authors declare that they have no conflict of interest.

\section{References}

1. L. Fellowfield, What is quality of life. http://www.medicine.ox.ac. uk/bandolier/painres/download/whatis/WhatisQOL.pdf Accessed 10 Nov 2018

2. L. Fellowfield, WHOQOL. Measuring quality of life, program on mental health. http://www.who.int/mental_health/media/68.pdf. Accessed 10 Nov 2018

3. Y. El Achhab, C. Nejjari, M. Chikri, B. Lyoussi, Disease-specific health-related quality of life instruments among adults diabetic: a systematic review. Diabetes Res. Clin. Pract. 80, 171-184 (2008)

4. J. Smith-Palmer, J.P. Bae, K.S. Boye, K. Norrbacka, B. Hunt, W. J. Valentine, Evaluating health-related quality of life in type 1 diabetes: a systematic literature review of utilities for adults with type 1 diabetes. Clin. Outcomes Res. 8, 559-571 (2016)

5. H.B. Nielsen, L.L. Ovesen, L.H. Mortensen, C.J. Lau, L.E. Joensen, Type 1 diabetes, quality of life, occupational status and education level - a comparative population-based study. Diabetes Res. Clin. Pract. 121, 62-68 (2016)

6. D.J. Wexler, R.W. Grant, E. Wittenberg, J.L. Bosch, E. Cagliero, L. Delahanty, M.A. Blais, J.B. Meigs, Correlates of health-related quality of life in type 2 diabetes. Diabetologia 49, 1489-1497 (2006)

7. H.M. Ledermann, Is maturity onset diabetes at young age (MODY) more common in Europe than previously assumed? Lancet 345, 648 (1995). 11

8. A.J. Chakera, A.M. Steele, A.L. Gloyn, M.H. Shepherd, B. Shields, S. Ellard, A.T. Hattersley, Recognition and Management of Individuals With Hyperglycemia Because of a Heterozygous Glucokinase Mutation. Diabetes Care 38, 1383-1392 (2015)

9. S. Bacon, M.P. Kyithar, S.R. Rizvi, E. Donnelly, A. McCarthy, M. Burke, K. Colclough, S. Ellard, M.,M. Byrne, Successful maintenance on sulphonylurea therapy and low diabetes complication rates in a HNF1A-MODY cohort. Diabet. Med. 33, 976-984 (2016)

10. B.M. Shields, S. Hicks, M.H. Shepherd, K. Colclough, A.T. Hattersley, S. Ellard, Maturity-onset diabetes of the young (MODY): how many cases are we missing? Diabetologia 53, 2504-2508 (2010)

11. M. Szopa, G. Osmenda, G. Wilk, B. Matejko, J. Skupien, B. Zapala, W. Młynarski, T. Guzik, M.T. Malecki, Intima-media thickness and endothelial dysfunction in GCK and HNF1AMODY patients. Eur. J. Endocrinol. 172, 277-283 (2015)

12. J. Skupien, S. Gorczynska-Kosiorz, T. Klupa, K. Cyganek, K. Wanic, M. Borowiec, J. Sieradzki, M.T. Malecki, Molecular background and clinical characteristics of HNF1A MODY in a Polish population. Diabetes Metab. 34, 524-528 (2008)

13. C. Bradley, C. Todd, T. Gorton, E. Symonds, A. Martin, R. Plowright, The development of an individualized questionnaire measure of perceived impact of diabetes on quality of life: the ADDQoL. Qual. Life. Res. 8, 79-91 (1999) 
14. C. Bradley, J. Speight, Patient perceptions of diabetes and diabetes therapy: assessing quality of life. Diabetes Metab. Res. Rev. 18 (Suppl 3), S64-S69 (2002)

15. C. Bradley, The audit of diabetes-dependent quality of life (ADDQoL). User guidelines. (Bradley, 2014) http://www.hea lthpsychologyresearch.com/Admin/uploaded/Guidelines/a ddqol18_userguidelines_rev24jan05a.pdf. Accessed 3 Apr 2014

16. M. Peyrot, R.R. Rubin, T. Lauritzen, F.J. Snoek, D.R. Matthews, S.E. Skovlund, Psychosocial problems and barriers to improved diabetes management: results of the Cross-National Diabetes Attitudes, Wishes and Needs (DAWN) Study. Diabet. Med. 22, 1379-1385 (2005)

17. N. Alcubierre, E. Rubinat, A. Traveset, M. Martinez-Alonso, M. Hernandez, C. Jurjo, D. Mauricio, A prospective cross-sectional study on quality of life and treatment satisfaction in type 2 diabetic patients with retinopathy without other major late diabetic complications. Health Qual. Life Outcomes 12, 131 (2014)

18. M.M. Collins, T. O'Sullivan, V. Harkins, I.J. Perry, Quality of life and quality of care in patients with diabetes experiencing different models of care. Diabetes Care 32, 603-605 (2009)

19. M. Sundaram, J. Kavookjian, J.H. Patrick, L.A. Miller, S.S. Madhavan, V.G. Scott, Quality of life, health status and clinical outcomes in Type 2 diabetes patients. Qual. Life. Res. 16, 165-177 (2007)

20. Y.T. Shim, J. Lee, M.P. Toh, W.E. Tang, Y. Ko, Health-related quality of life and glycaemic control in patients with Type 2 diabetes mellitus in Singapore. Diabet. Med. 29, e241-e248 (2012)

21. W.K. Redekop, M.A. Koopmanschap, R.P. Stolk, G.E. Rutten, B. H. Wolffenbuttel, L.W. Niessen, Health-related quality of life and treatment satisfaction in Dutch patients with type 2 diabetes. Diabetes Care 25, 458-463 (2002)

22. M. Sundaram, J. Kavookjian, J.H. Patrick, Health-related quality of life and quality of life in type 2 diabetes: relationships in a cross-sectional study. Patient 2, 121-133 (2009)

23. M. Donald, J. Dower, J.R. Coll, P. Baker, B. Mukandi, S.A. Doi, Mental health issues decrease diabetes-specific quality of life independent of glycaemic control and complications: findings from Australia's living with diabetes cohort study. Health Qual. Life Outcomes 11, 170 (2013)

24. S.S. Soon, S.Y. Goh, Y.M. Bee, J.L. Poon, S.C. Li, Audit of Diabetes-Dependent Quality of Life (ADDQoL) [Chinese Version for Singapore] questionnaire: reliability and validity among Singaporeans with type 2 diabetes mellitus. Appl. Health Econ. Health Policy 8, 239-249 (2010)

25. J.O. Chung, D.H. Cho, D.J. Chung, M.Y. Chung, An assessment of the impact of type 2 diabetes on the quality of life based on age at diabetes diagnosis. Acta Diabetol. 51, 1065-1072 (2014)

26. A.M. Steele, B.M. Shields, K.J. Wensley, K. Colclough, S. Ellard, A.T. Hattersley, Prevalence of vascular complications among patients with glucokinase mutations and prolonged, mild hyperglycemia. JAMA 311, 279-286 (2014) 